Questions de communication

$24 \mid 2013$

Renouvellement des mises en scène télévisuelles de la politique

\title{
Anna SAM, Mon tour de France des blogueurs
}

Paris, Stock, coll. Essais et documents, 2011, 216 pages

\section{Gilles Boenisch}

\section{OpenEdition}

\section{Journals}

Édition électronique

URL : http://journals.openedition.org/questionsdecommunication/8848

DOI : 10.4000/questionsdecommunication. 8848

ISSN : 2259-8901

\section{Éditeur}

Presses universitaires de Lorraine

Édition imprimée

Date de publication : 31 décembre 2013

Pagination : $322-323$

ISBN : 978-2-8143-0182-5

ISSN : 1633-5961

\section{Référence électronique}

Gilles Boenisch, "Anna sam, Mon tour de France des blogueurs », Questions de communication [En ligne], 24 | 2013, mis en ligne le 01 février 2014, consulté le 22 septembre 2020. URL : http:// journals.openedition.org/questionsdecommunication/8848 ; DOl : https://doi.org/10.4000/ questionsdecommunication.8848 
la technologie, le design, l'art, les médias, être amateur de jeux, être un grand collaborateur et être intéressé par la façon dont les choses fonctionnent » (accès : http://q21.org/admissions; consulté le 24/I0/I3).

Français et Anglo-saxons ne parlent pas du même objet lorsquills discutent ensemble des vertus du jeu et de son introduction dans tous les actes de la vie, notamment dans la pédagogie. Par « jeu », les premiers entendent les règles et le plaisir que le joueur en retire; les seconds ne s'intéressent qu'au contenu et aux compétences qu'il permet d'acquérir. Les jeux ne peuvent gagner tous les aspects de notre vie sans risquer de devenir une industrie et de masquer des intérêts de marketing. Sans création collaborative, les jeux, en particulier ceux à réalité alternée, perdent tout leur sens, celui de transformer le devenir perpétuel des sociétés techniques en un avenir choisi qui réintroduit le « nous » dans l'interfaçage avec les réseaux. Ce « nous » ne peut émerger que si les citoyens sont associés à l'élaboration de l'œuvre et invités à quitter leur posture de consommateur pour celle de créateur. Ces jeux doivent conserver une construction non téléguidée où chaque participant façonne un aspect de l'œuvre pour devenir une médiation dans le sens que lui donne Jean Caune (Pour une éthique de la médiation. Les sens des pratiques culturelles, Grenoble, Presses universitaires de Grenoble, 1999) de relation singulière du sujet à autrui et au monde.

Françoise Lejeune

CREM, université de Lorraine, 57000 francoiselejeune@yahoo.fr

\section{Anna SAM, Mon tour de France des blogueurs.}

Paris, Stock, coll. Essais et documents, 201 I, 216 p.

Inauguré par un petit nombre de pionniers, puis adopté par un public de plus en plus large, le blog a connu un essor planétaire fulgurant. Apparu au Canada en 1990, il devient populaire dès 2000, pour devenir un incontournable. II désigne un site internet réactualisé fréquemment, animé par une personne ou un groupe et transmettant des informations et des opinions sur un certains nombre de sujets. Le terme est l'abréviation de weblog, qui signifie « journal de bord ». Jour après jour, y sont notés les événements importants. La majorité des blogs sont issus de particuliers qui les utilisent à des fins d'autoreprésentation. Cela s'explique par la simplicité de mise en œuvre et, comme pour les réseaux sociaux et les wikis, par le besoin d'affirmer sa présence numérique et de participer.Pourtant, malgré leur grande popularité qui a profondément modifié la pratique de publication d'un journal en ligne, les blogs ne sont pas toujours bien appréhendés. Mon tour de France des blogueurs décrit le statut de ces espaces d'expression personnels, dix ans après leur apparition. En dehors de certains blogs populaires, on ignore qui se cache derrière les contenus, les commentaires, les univers construits qu'ils représentent. Pour Anna Sam, les blogs seraient « une nouvelle manière de fabriquer nos sociétés » (quatrième de couverture), un véritable phénomène sociétal devant essentiellement son succès à sa liberté éditoriale et à sa capacité d'interaction en temps réel. Même s'il existe un décalage entre les générations qui n'éprouvent pas toutes de l'engouement pour les outils numériques, la tendance du blog s'étend des jeunes générations aux seniors n'hésitant plus à partager avec le plus grand nombre. Faisant elle-même partie de cette communauté, Anna Sam s'intéresse à ces personnes qui passent leur temps à mettre à jour leurs pages, pour savoir qui ils sont, comment ils gèrent cette activité qui se superpose à leur vie personnelle et professionnelle, ou encore pour savoir comment leurs notoriétés sur le web à influencé leurs parcours: «Qui sont donc ces hommes et ces femmes qui, un beau jour, ont décidé de faire du web le refuge de l'égalité de parole? » (p. 12).

L'essai invite donc à découvrir quinze blogueurs français à travers une série de portraits disparates. Bande dessinée, œnologie, mode, cuisine, nouvelles technologies ou publicité, il y a autant de sujets que de blogueurs, « l'échantillon est aussi riche que les personnes qui les incarnent » (p. 18). Ceux-là ont donc confié leur intimité et la genèse de leurs activités numériques en explicitant la passion qui les anime. Pour l'auteure, il s'agit d'aller au-delà de l'image dématérialisée créée pour la publication en ligne en s'interrogeant sur le sens que produit cette activité (p. 14). Elle rappelle que le blog est la seule activité qui a « survécu à toutes les modes 》 (p. 15) des nouveautés éditoriales du réseau, malgré la domination des réseaux sociaux et toutes les déclinaisons liées à l'internet mobile. Cette longévité s'explique probablement par la spécificité du blog, fournir un espace personnel, non nécessairement professionnel, gratuit, donnant la possibilité de s'exprimer sur le réseau, de partager, de susciter des rencontres.

« ll y a un coté un peu égocentrique [... ] mais vertueux, ça pousse les gens à faire toujours mieux. Les notions de partage, de durée sont omniprésentes. On y trouve une compétition très positive. Le niveau de qualité 
de connaissances est en pleine expansion. Ceux qui bloguent entrent dans une démarche réfléchie même si, au début, c'est assez instinctif. II reste cependant toujours un but : entretenir une conversation avec la communauté et produire quelque chose qui donne envie aux lecteurs de revenir » (p. I5).

Malgré cette diversité, chacun s'accorde sur l'importance des « posts » et la possibilité qu'a le lecteur de commenter chaque billet, qui modifie considérablement le pacte autobiographique initial : « Le contenu, c'est le business d'anonymes passionnés, même si une poignée d'entre eux accède par la suite au rang convoité de blogueurs professionnels. Les outils mis à disposition, simples, accessibles, s'effacent alors pour laisser la place au pur contenu qui marquera la différence entre blogueurs » (pp. 15-16).

Les points communs ressortant de ces portraits sont la générosité, le partage, la curiosité, quelle que soit la thématique traitée. En cela, les blogs génèrent rencontres fortuites, rapprochements, affinités, communautés, mêlant de multiples attentes, relevant toutes de l'enrichissement personnel et social. En effet, pour les blogueurs, coexistent de multiples raisons de trouver dans les réactions des lecteurs une forme de reconnaissance identitaire. Ce que l'on comprend de cette réappropriation du web par les internautes qui deviennent autant producteurs que consommateurs d'informations, c'est la complexification de la sphère informative par le jeu de «l'intimité construite » et exposée au public, « l'idée idéalisée d'une vie ouverte en temps réel » (pp. 70-177).

Cette observation atypique est révélatrice de la diversité des répertoires d'usages, des dynamiques et pratiques sociales, face à l'introduction du blog comme objet communicationnel nouveau et massivement adopté. Ainsi la « blogosphère » fait-elle coexister différents groupes, dotés de références qui conditionnent les choix et affinités. II s'agit d'une sorte de spatialisation de pratique sociale, fait d'un assemblage de doubles sociaux virtuels qui vont développer, moduler et expérimenter de nouvelles personnalités où s'élaborent des pratiques sociotechniques qui semblent viser des fins d'émancipation, d'accomplissement ou de sociabilité, subjective, individuelle et collective. II en résulte un texte singulier et marqué qui encourage une résonance théorique tirée des multiples témoignages exploitant le ton et le style des blogs. Se dégage de cette démarche une réelle dimension critique sur le texte lui-même, qui engage une seconde lecture, plus minutieuse, donnant aux expressions une grande profondeur analytique.
Enfin, la Commission générale de terminologie et de néologie a publié au Journal officiel (20/05/05) un avis établissant une liste de termes et d'expressions destinés à supplanter les anglicismes sur l'internet. Ainsi « blocnotes $»$, que l'on peut accepter sous sa forme abrégée « bloc », désigne-t-il « un site sur la Toile, souvent personnel, présentant en ordre chronologique de courts articles ou notes, généralement accompagnés de liens vers d'autres sites 》. Ce que nous continuons d'appeler un blog.

Gilles Boenisch

CREM, université de Lorraine, F-57000 gilles.boenisch@gmail.com 